Journal of Computer Science 5 (6): 440-444, 2009

ISSN 1549-3636

(C) 2009 Science Publications

\title{
Novel Method of the Combination of Forecasts Based on Rough Sets
}

\author{
Eslam Faik Ahmed, Wang Jia Yang and Maan Younis Abdullah \\ School of Information Science and Technology, \\ Central South University, Changsha, China
}

\begin{abstract}
Problem statement: A study in Analytic Hierarchy Process (AHP) had shown the problem of quantify the qualitative and the side Combined. Approach: So that problems were better resolved. The rough sets theory and AHP was introduced in the study, furthermore, these were united to create a completely new method of combination forecasts. Results: The results of numerical examples were shown to illustrate the interval AHP models reflecting the uncertainty of evaluations in nature. Conclusion: Therefore our method can be analyzed in order to make the best decision-making and makes combination forecast more objective. Further, the proposed procedure generates a set of easily understood rules that can be readily applied in knowledge-based.
\end{abstract}

Key words: Rough set, Combination forecasting, AHP

\section{INTRODUCTION}

With the development of human society, its uncertain extent increasingly goes highly. We have to face more and more complex systems. It's much more difficult for us to make decisions thanks to the highly complexity of systems. Consequently, confronted with complex systems, people pay more attention to research and apply methods as scientifically as possible to forecast and make decisions.

The theory of rough sets deals with the approach of an arbitrary subset of a universe by two definable or observable subsets called lower and upper approximations ${ }^{[1]}$. There are at least two methods for the development of this theory, the constructive and algebraic approaches. In constructive methods, lower and upper approximations are not primitive notions. They are assembled from other concepts, such as binary relations on a universe ${ }^{[1]}$, partitions and coverings of a universe and partially ordered sets ${ }^{[2]}$, lattice, Boolean algebras and their sub algebras ${ }^{[3-5]}$. On the other hand, by treating lower and upper approximations as primitive notions, algebraic (axiomatic) methods focus on algebraic systems for the theory of rough sets. A set of axioms is used to characterize approximation operators that are the same as the ones produced using constructive methods ${ }^{[6]}$.

Pawlak and scholars from Poland in ${ }^{[7,9,10,11,]}$, made of rough set theory is highly abstract logic of human simulation Functions. The main idea is the ability to keep classified information system under the premise of change through knowledge reduction, the issue of export must Classification rules or policies. AHP method developed by Saaty in the initial stage of the seventies of the twentieth century as a method of decision-making. In the intricate Complex circumstances, people expect to make optimal use of information in decision-making, such as the employer's selection of personnel, for example the workers in the company how divide the work to the employer. AHP is a quantitative characterization of the problem into line the effective method. However, AHP also has its own limitation, that is the percentage of subjective factors too, the results sometimes unconvincing.

In this study, the use of AHP to the results of the use of rough set theory based on a combination of the forecast to Law, will determine the weights of importance into the issue of property by a simple quantitative terms, the various indicators to be important, the indemnity AHP meeting brought about by the subjective bias, so that the credibility of a more objective selection.

Basic concepts of the rough sets: Information system and indiscernibility relation formally, an information system, can be seen as a system:

$$
\mathrm{IS}=(\mathrm{U}, \mathrm{A})
$$

Where:

$\mathrm{U}=$ The universe (a finite set of objects, $\mathrm{U}=\{\mathrm{x} 1$, $\mathrm{x} 2, \ldots ., \mathrm{xm}\})$

$\mathrm{A}=$ The set of attributes

Corresponding Author: Eslam Faik Ahmed, School of Information Science and Technology, Central South University, Changsha, China 
Each attribute $\mathrm{a} \in \mathrm{A}$ defines an information function fa: $U \rightarrow V a$, where $V a$ is the set of values of a, called the domain of attribute a. For every set of attributes $\mathrm{B} \subset \mathrm{A}$, an indiscernibility relation $\operatorname{Ind}(\mathrm{B})$ is defined in the following way: two objects, $\mathrm{Xi}$ and $\mathrm{Xj}$, are indiscernible by the set of attributes $B$ in $A$, if $b(X i)=b(X j)$ for every $b \subset B$. The equivalence class of $\operatorname{Ind}(B)$ is called elementary set in B because it represents the smallest discernible groups of objects. For any element Xi of U, the equivalence class of $\mathrm{Xi}$ in relation $\operatorname{Ind}(\mathrm{B})$ is represented as) [Xi]Ind(B). The construction of elementary sets is the first step in classification with rough sets.

Figure 1 Approximations of sets so called the lower and the upper approximations of a set, referring to:

Let $\mathrm{X}$ denotes the subset of elements of the universe $\mathrm{U}(\mathrm{X} \subset \mathrm{U})$. The lower approximation of $\mathrm{X}$ in $\mathrm{B}$ $(\mathrm{B} \subseteq \mathrm{A})$, denoted as:

$\mathrm{BX}=\{\mathrm{Xi} \in \mathrm{U} \mid[\mathrm{Xi}] \operatorname{Ind}(\mathrm{B}) \subset \mathrm{X}\}$

The upper approximation of the set $\mathrm{X}$, denoted as:

$\overline{\mathrm{BX}}=\{\mathrm{Xi} \in \mathrm{U} \mid[\mathrm{Xi}] \operatorname{Ind}(\mathrm{B}) \cap \mathrm{X} \neq \phi\}$

For any object of the lower approximation of $\mathrm{X}(\mathrm{i} . \mathrm{e} ., \mathrm{Xi} \in \mathrm{BX})$, it is certain that it belongs to $\mathrm{X}$. For any object $\mathrm{Xi}$ of the upper approximation of $\mathrm{X}$ (i.e., $\mathrm{Xi} \in \overline{\mathrm{BX}}$ ), we can only say that $\mathrm{Xi}$ may belong to $\mathrm{X}$. The difference: $\mathrm{BNX}=\overline{\mathrm{BX}}-\mathrm{BX}$ is called a boundary of $\mathrm{X}$ in $\mathrm{U}$.

If the lower and upper approximation are Identical (i.e., $\overline{\mathrm{BX}}=\mathrm{BX}$ ), then set $\mathrm{X}$ is definable, otherwise, set $\mathrm{X}$ is indefinable in $\mathrm{U}$. If $\mathrm{BX} \neq \phi$ and $\overline{\mathrm{BX}} \neq \mathrm{U}, \mathrm{X}$ is called roughly definable in $U$; where $\Phi$ denotes an empty set.

And POSB $(\mathrm{X})=\mathrm{BX}$ called the $\mathrm{B}$-positive region of $X, \operatorname{NEGB}(X)=U-B X$, called the $B$-negative region of $X$.

The weight ac approximation of sets: An accuracy measure of the set $\mathrm{X}$ in $\mathrm{B} \subseteq \mathrm{A}$ is defined as:

$\mu_{\mathrm{B}}(\mathrm{X})=\operatorname{card}(\underline{\mathrm{BX}}) / \operatorname{card}(\overline{\mathrm{BX}})$

where, card (.) means the cardinality of a set. As one can notice, $\left(0 \leq \mu_{\mathrm{B}}(\mathrm{X}) \leq 1\right)$.

If $X$ is definable in $U$.

Then:

$\mu_{\mathrm{B}}(\mathrm{X})=1$

If $\mathrm{X}$ is un-definable in $\mathrm{U}$, then:

$\mu_{\mathrm{B}}(\mathrm{X})<1$

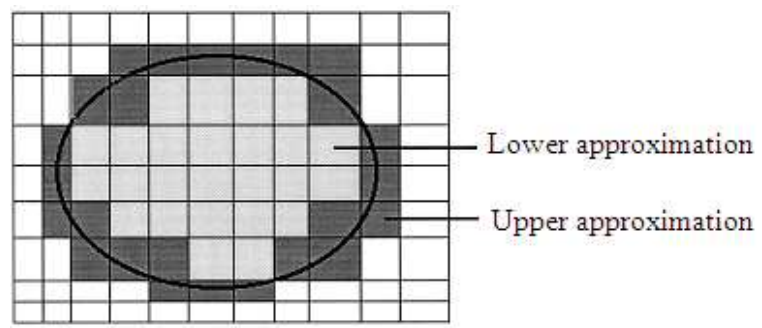

Fig. 1: Schematic demonstration of the upper and lower approximation of set $\mathrm{X}$

Reduction and Independence of attributes: If Ind (A) = Ind (A-ai), then the attribute ai is called superfluous. Otherwise, the attribute ai is indispensable in A.

If the set of attributes is dependent, one can be interested in finding all possible minimual subsets of attributes, which lead to the same number of elementary sets as the whole set of attributes (reducts) and in finding the set of all indispensable attributes (core).

The concepts of core and reduct are two fundamental concepts of the rough sets theory. The core is the common part of all reducts. To compute reducts and core, the discernibility matrix is used. The discernibility matrix has the dimension $\mathrm{n} \times \mathrm{n}$, where $\mathrm{n}$ denotes the number of elementary sets and its elements are defined as the set of all attributed which discern elementary sets $[\mathrm{X}]_{\mathrm{i}}$ and $[\mathrm{X}]_{\mathrm{j}}$ :

Basic idea of AHP: When people on the issue of lack of data in decision making, It is often used $\mathrm{AHP}^{[7]}$ to quantify the qualitative and the side Combined, so that problems are better resolved, the level of analysis of the basic idea are decomposition equivalent and comprehensive calculated on each floor in each of the combined weights of indicators, to be compared with the overall objective of the priority

\section{MATERIALS AND METHODS}

Figure 2, we test volunteers to fill an explanation as an example. The assumption that a choice of the workers and divide pursuant to expert. This example taken form any companies system available to choose from seven sections, the company choose the worker who have expert and as indicated in the $\mathrm{CV}$ are as follows are: The duration of the experience, the employment situation, employment hardware conditions, manager ,research funding and admission scores .

In Table 1 of six before the data is based on six factors experts comprehensive quantitative and score; the last one $(\mathrm{Y})$ in the number of according to there are 
seven jobs in the rankings. Score lower, on behalf of the job of the more satisfied. The next question is: To compare the $\mathrm{n}$ factor $\mathrm{B} 1, \ldots, \mathrm{Bn}$ the impact on the target layer two by two attributes were compared with the method of the importance of the various factors of the qualitative part of the number, as a paired comparison matrix. Pair wise comparison matrix, as there is obviously from 1-9, between the numerical as the scale, significance is as in Table 2.

In a paired comparison of the consistency test array, the next $\mathrm{n}$ months of this study is an important factor in carrying out a scheduled sequence. Often used method is based on paired comparison of the largest proper value matrix $\lambda \max$ corresponding features vector: $\mathrm{W}=\left(\mathrm{w}_{1} \ldots \mathrm{w}_{\mathrm{n}}\right)$ as factor $\mathrm{n}$ of the weight vector.

Based on rough set theory combined forecasting method $^{[8]}$; Known object-based prediction of $y$ of $n$ historical data $\mathrm{y}_{\mathrm{t}}(1 \leq \mathrm{t} \leq \mathrm{n})$, choose the $\mathrm{m}$ different types of forecasting methods $\mathrm{c}_{1}, \ldots, \mathrm{c}_{\mathrm{m}}$. To predict, set up the first $i$ of forecasting methods in the first $t$ value of the forecast period cit, $\beta(1 \leq i \leq m)$ for the first $i$ of the weight coefficient prediction method, $t$ the period of pre-value, please take a weighted average of $c_{i t}$ :

$\sum_{i=0}^{m} \beta_{i} c_{i t}$

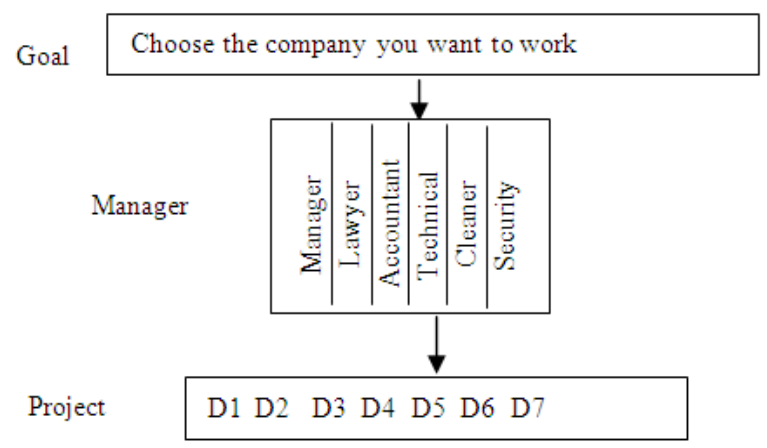

Fig. 2: Worker as indicated in the CV

\begin{tabular}{lrrrrrrr}
\multicolumn{7}{l}{ Table 1: The relevant information } \\
\hline \multicolumn{1}{l}{ B1 } & B2 & B3 & B4 & B5 & B6 & Y \\
\hline D1 & 10 & 8 & 28 & 23 & 23 & 10 & 2 \\
D2 & 9 & 15 & 13 & 14 & 51 & 9 & 5 \\
D3 & 17 & 14 & 26 & 12 & 5 & 17 & 1 \\
D4 & 16 & 12 & 44 & 17 & 10 & 16 & 7 \\
D5 & 14 & 13 & 27 & 16 & 9 & 14 & 3 \\
D6 & 19 & 10 & 31 & 18 & 14 & 19 & 6 \\
D7 & 21 & 7 & 19 & 25 & 19 & 21 & 4 \\
\hline
\end{tabular}

Table 2: The numerical scales significance

\begin{tabular}{llllll}
\hline $\begin{array}{l}\text { From bi } \\
\text { To bi }\end{array}$ & Same & $\begin{array}{l}\text { Some } \\
\text { important }\end{array}$ & Important & $\begin{array}{l}\text { Very } \\
\text { important }\end{array}$ & $\begin{array}{l}\text { Absolutely } \\
\text { important }\end{array}$ \\
\hline $\mathrm{a}_{\mathrm{ij}}$ & 1 & 3 & 5 & 7 & 9 \\
\hline
\end{tabular}

In order to determine the weight coefficient $\beta$, various forecasting methods, we will be regarded as condition attributes, then $\mathrm{C}=\left(\mathrm{c}_{1} \cdot \mathrm{c}_{\mathrm{m}}\right)$, indicators to predict object $\mathrm{Y}$ as decision attribute, then $\mathrm{D}=(\mathrm{y})$, the first $t$ periods of the various forecasting methods and forecasting predictive value of the historical data table object that for ut $=\left(c_{1 t}, \ldots, c_{m t} ; y_{t}\right)$, so on the domain $\mathrm{U}=\left(\mathrm{u}_{1}, \ldots, \mathrm{u}_{\mathrm{n}}\right) \cdot \mathrm{U}_{\mathrm{t}}$ posed by the information table is the combination of two-dimensional square forecast law model of the relationship between the data tables. Thus, weights can be used as follows $\beta$ rough set theory-based approach to determine:

Step 1: According to the definition of 3, calculated $R_{D}$ of knowledge dependence on $\mathrm{R}_{\mathrm{C}}$.

Step 2: Prediction method for each ci, the calculation $\mathrm{RD}$ knowledge of RC (i) the dependence on:

$\left\{(\mathrm{u}, \mathrm{v}) \in \mathrm{U} * \mathrm{U} \mid \mathrm{c}_{\mathrm{j}}(\mathrm{u})=\mathrm{c}_{\mathrm{j}}(\mathrm{v}), \forall \mathrm{c}_{\mathrm{j}} \neq \mathrm{c}_{\mathrm{i}}\right\}$

Recorded as $\mathrm{R}^{\mathrm{C}}(\mathrm{i}), \mathrm{i}=1, \ldots, \mathrm{m}$.

Step 3: Calculation of SIG (ci, C, D).

Step 4: If the SIG (ci, C, D) $=0$, delete ci; when SIG $(\mathrm{ci}, \mathrm{C}, \mathrm{D}) \neq 0$, So:

$$
\beta_{i}=\frac{\operatorname{SIG}\left(c_{i}, C, D\right)}{\sum_{i=1}^{m} \operatorname{SIG}\left(c_{i}, C, D\right)}
$$

Combination of rough set theory and AHP forecasting methods: Analysis of a model-level paired comparison matrix at all levels of calculation; the general could be the result of the following Quasi -the layer, the factors of B1 ... Bn weight vector, for each element $\mathrm{Bi}$, program layer by various $\mathrm{Dj}$ the corresponding weight vector, respectively and finally, each program the corresponding weighted

Rough set theory and then use the decision Table 3 structure, use of the above three outcomes to this decision-making Table 3 the results (3): As a New condition attributes, from the results of (2): In each of the $B_{i, j}$ structures the decision-making table of the attribute value $u_{t}$ object cit. This combination of pre-use can be re-measured to calculate the weight of each factor, each of the last to be weighted and the new.

\begin{tabular}{llllll}
\multicolumn{2}{l}{ Table 3: The decision } \\
\hline Domain object & C3 & C4 & C6 & C7 & y \\
\hline u1 & 0.1208 & 0.1047 & 0.0734 & 0.1068 & 0.2143 \\
u2 & 0.2602 & 0.1753 & 0.1584 & 0.1829 & 0.1071 \\
u3 & 0.1293 & 0.1983 & 0.0146 & 0.1354 & 0.2500 \\
u4 & 0.0760 & 0.1424 & 0.3115 & 0.1733 & 0.0357 \\
u5 & 0.1263 & 0.1485 & 0.1060 & 0.1332 & 0.1786 \\
u6 & 0.1080 & 0.1342 & 0.0845 & 0.1114 & 0.0714 \\
u7 & 0.1794 & 0.0955 & 0.2417 & 0.1571 & 0.1429 \\
\hline
\end{tabular}




\section{J. Computer Sci., 5 (6):440-444, 2009}

\section{RESULTS}

The model based on the importation of the first layer, respectively, comparison matrix A6×6 (6 factors moments paired comparison Array) and seven worker in each factor of the paired comparison matrix under the $\mathrm{M} 1, \ldots, \mathrm{M} 6$ (they are $7 \times 7$ matrix bands):

$$
\mathrm{a}=\left[\begin{array}{cccccc}
1 & 4 & 1 / 5 & 1 / 8 & 1 & 1 / 6 \\
1 / 4 & 1 & 1 / 8 & 1 / 9 & 5 & 1 / 8 \\
5 & 8 & 1 & 1 / 3 & 5 & 1 / 2 \\
8 & 9 & 3 & 1 & 4 & 3 \\
1 & 3 & 1 / 5 & 1 / 4 & 1 & 1 / 6 \\
6 & 8 & 2 & 1 / 3 & 6 & 1
\end{array}\right]
$$

In Table 3 a calculation by the maximum proper value and corresponding proper value of the following six factors to be this weight vector: $\mathrm{B}=(0.0760 .023$ $\begin{array}{llll}0.233 & 0.329 & 0.066 & 0.274)\end{array}$.Weight vector to be in accordance with their rankings: $\mathrm{B} 4>\mathrm{B} 6>\mathrm{B} 3>\mathrm{B} 1>\mathrm{B} 5>$ B2. M1,..., M6 proper value corresponding to the six are as follows:

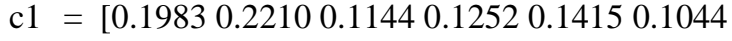

$$
\begin{aligned}
& \text { 0.0952] }
\end{aligned}
$$

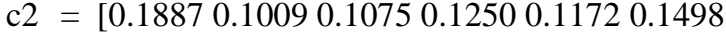

$$
\begin{aligned}
& 0.2108] \\
& \text { c3 }=\left[\begin{array}{lllll}
0.1208 & 0.2602 & 0.1293 & 0.0760 & 0.12630 .1080
\end{array}\right. \\
& 0.1794]
\end{aligned}
$$

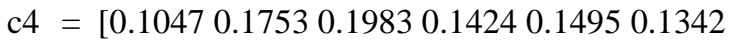

$$
\begin{aligned}
& 0.0955]
\end{aligned}
$$

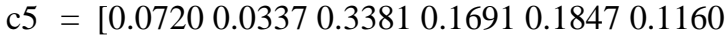

$$
\begin{aligned}
& 0.0865] \\
& \text { c6 }=\left[\begin{array}{lllll}
0.0734 & 0.1584 & 0.0246 & 0.31150 .1060 & 0.0845
\end{array}\right. \\
& \text { 0.2417] }
\end{aligned}
$$

In the calculation of the six paired comparison matrix M1,..., M6 corresponding proper value, the seven worker have been factors in each of The right value, then the total score vector for each department:

$$
\mathrm{C}=\begin{aligned}
& (0.1068,0.1829,0.1354,0.1733,0.1332,0.1114, \\
& 0.1571)
\end{aligned}
$$

Therefore, AHP obtained by the seven top workers (Previous post): D2, D4, D7, D3, D5, D6 and D1, due to factors "admission", the scores of high worker with low value, so the ranking results and the known results can be found in some differences in subjective factors can be seen in the level of analysis or be affected.

Next, to this company as a 7 departments: $\mathrm{u} 1, \ldots, \mathrm{u} 7$. According to the calculation of the weight vector $\mathrm{B}$, the six factors selection of the most important three factors B3, B4, B6, seven worker in the three factors of the weight vector prediction method for $\mathrm{c} 3, \mathrm{c} 4$ And $\mathrm{c} 6$ and then calculated the earlier of the weight vector $\mathrm{C}$ is also seen as a prediction method $\mathrm{c} 7$, the value of predictor (y) represented company rankings standardization. In this way, a decision-making table is produced.

\section{DISSCUSION}

In accordance with the following information to calculate the properties of entropy weights will be related to various attributes of the calculation of equivalence classes. Type of method is to attribute the characteristics of value ${ }^{[8]}$, that attribute for each attribute value divided by the characteristics of a number of characteristic values. We have two of each domain of the distance between the attribute values (maximum norm) to determine they are a class of all. For example, to determine $U \mid R_{C}$, the distance between every two attribute values within 7 neighborhood domains (u1, u2), (u1, u3) ... (u6, u7), a total of 21 values, followed by their average calculated.

Finally, in Table 4. the end to determine which are objects can be used as class. With such an approach can be: $\mathrm{U}\left|\mathrm{R}_{3}^{\mathrm{c}}, \ldots \mathrm{U}\right| \mathrm{R}_{7}^{\mathrm{c}}$ four Price category. With the equivalence class information entropy can be used on the relevant formula to calculate the weights of attributes. The result of Calculation: $\mathrm{H} 0\left(\mathrm{R}_{\mathrm{D}} \mid \mathrm{R}_{\mathrm{C}}\right)=0.1070$. By the combination forecasting model:

$\gamma=\sum_{\mathrm{i}=0}^{\mathrm{m}} \beta_{\mathrm{i}} \mathrm{c}_{1 \mathrm{t}}$

The seven workers received the new rankings (previous post): D4, D7, D2, D5, D6, D3, D1. The results of AHP and the calculation results do have some differences, but a closer look can be found: 7 workers ranking position in general has not changed, that is similar to the difference in the ranking of several workers and no ranking is a big gap between the two swap the location of the company situation. As a result of the calculation in the combination of forecasts will be the level of analysis as the final result is a pre-measurement methods and reference information has been forecast as a result, naturally derived from its position over the final level of analysis alone is more for the comprehensive and objective.

Table 4: The importance of forecasting methods and weights

\begin{tabular}{lllll}
\hline & C4 & C6 & C7 & \\
\hline Dependence & 0.0970 & 0.1379 & 0.0408 & 0.0662 \\
importance of & 0.0700 & 0.2157 & 0.4634 & 0.2857 \\
weights & 0.0676 & 0.2084 & 0.4479 & 0.2760 \\
\hline
\end{tabular}


On the other hand, through the use of rough set theory and the calculation of information entropy and prediction method to overcome the combination of the original Subjectivity. Therefore, this new position better reflects the actual situation, it is also useful.

\section{CONCLUSION}

Rough set theory in decision-making table attributes that the information entropy can measure the importance of attributes and attribute reduction. Level although the analysis can be effectively translated into qualitative quantitative analysis of the issue of decisionmaking, but the influence of subjective judgments.

In this study, the results of AHP were constructed to characterize the decision-making table, combination forecasting method with the weights of attributes. It should be noted that: method of data classification will have a direct impact on the results. Therefore, both the characteristics of the side or the other method can be further analyzed in order to make the best decisionmaking.

Through this way we can easily turn the fact that models are established automatically into truth. And the module will choose an all set that has integrated all kinds of knowledge to settle complex problems, which now is in study. It's believed that it will be widely developed and applied in future.

\section{REFERENCES}

1. Pawlak, Z., 1982. Rough sets. Int. J. Comput. Inform. $\quad$ Sci., $\quad 11$ 341-356. http://www.springerlink.com/content/r5556398717 $921 \times 5$

2. Yao, Y.Y. and T.Y. Lin, 1996. Generalization of rough sets using modal logic. Intell. Automat. Soft Comput. Int. J., 2: 103-120. http://citeseerx.ist.psu.edu/viewdoc/summary?doi= 10.1.1.48.7933
3. Vander Aalst, W., M. Weske and D. GrÄunbauer, 2005. Case handling: A new paradigm for business process support. Data Knowl. Eng., 53: 129-162. http://bpt.hpi.uni-

potsdam.de/pub/Public/MathiasWeske/sdarticle.pdf

4. Gehrke, M. and E. Walker, 1992. On the structure of rough sets. Bull. Polish Acad. Sci. Math., 40: 235-245. http://www.math.nmsu.edu/ elbert/roughsetsswp5 $0 . p d f$

5. Cattaneo, G., 1996. Mathematical foundations of roughness and fuzziness. Proceedings of the 4th International Workshop on Rough Sets, Fuzzy Sets and Machine Discovery, (RSFSMD'96), University of Tokyo, Tokyo, pp: 241-247.

6. Yao, Y.Y., 1996. Two views of the theory of rough sets in finite universes. J. Approximate Reason., 15: 291-317.

http://citeseerx.ist.psu.edu/viewdoc/summary?doi= 10.1.1.54.5584

7. Rubinstein, Z. and D. Corkill, 2003. Mixedinitiative management of dynamic business processes. Proceedings of the 2003 IEEE International Workshop on Soft Computing in Industrial Applications, June 23-25, IEEE Xplore Press, Binghamton, New York, USA., pp: 39-44. DOI: 10.1109/SMCIA.2003.1231341

8. Zhong, Bo. and X. Zhi, 2002. A compound projection method based on coarse aggregate theory. Statistical Research., 11: 37-39. http://scholar.ilib.cn/A-tongjyj200211009.html

9. ShiFeng, Z., L. Liang and Z. Yong Qing, 2002. An improved rough set attribute reduction heuristic algorithms. Shanghai Jiaotong Univ., 36: 478-481.

10. Deng, F., 2002. Rough set theory and its application in multi-objective optimization. Xi'an University of Electronic Science and Technology Department of Applied Mathematics, Xi'an.

11. Hongyuan, 1997. Mathematical Modeling. 1st Edn., East China Normal University Press, Shanghai, ISBN: 7-5617-1588-9, pp: 306. 